\title{
Cardiovascular imaging in South Africa
}

'Humanity has but three enemies: Heart Failure, Famine and War; by far the greatest, by far the most terrible, is Heart Failure.' [Sir William Osler]

\section{Background}

The role of the radiologist in cardiovascular imaging has followed a tortuous and often uphill road worldwide, with certainly no exception in South Africa. Since the discovery of X-rays by Röntgen in 1895, the turf war between clinicians and radiologists regarding their ownership has always been fiercest around the imaging of the heart and great vessels.

I vividly remember the meetings, during my pre- and post-graduate training years, between radiologists, cardiologists and thoracic surgeons, at which coronary angiograms, chest X-rays, CT scans (in their infancy) and, much later, echocardiograms were discussed. Input from radiologists was valued during these discussions. This interdisciplinary contact has dwindled and petered out in most academic and private institutions. Nowadays, a conventional coronary angiogram rarely finds its way into the X-ray department.

Initially, most echocardiograms were performed by radiologists, but this procedure rapidly settled snugly inside the cardiologist's consulting room and became part of his or her routine examination. The radiologist or sonographer currently plays a minor role: mainly to exclude or confirm the presence of congenital, valvular or functional abnormalities referred by general practitioners, paediatricians, and others.

The birth of multislice CT, especially 32 - and 64-slice scanners, sparked renewed enthusiasm among radiologists to delve into the coronary vessels (which had become the sole domain of the cardiologist). This occurrence did not go unnoticed among the cardiology community, resulting in them purchasing their own CT equipment - leading to a renewed turf battle between the two groups. But because of the ominous potential for self-referral (resulting in escalating costs), CTA studies remained in the hands of radiologists.

Despite arguments pointing to the fact that a non-invasive procedure might increase the detection of important lesions and increase referrals for intervention (and in the process decrease unnecessary invasive coronary angiograms), referrals from cardiologists remained low.

In addition to a notable professional and academic career (and provincial colours for squash and swimming), Leonie Scholtz established her own radiology practice in 1993, which has subsequently expanded to 8 partners and 15 practices in Pretoria and the Lowveld. She has authored and co-authored numerous articles published in peer-reviewed and accredited journals, and has been an invited speaker at national and international conferences and workshops. She was Professor and Head of the Department of Diagnostic Radiology and Nuclear Medicine at the University of Pretoria from 2002 - 2005, and vice-president of the Radiological Society of South Africa from 2004 - 2007. Her interests and pastimes include art, photography and cinematography, French and English literature, and cycling.
Taking into consideration that this was a unique situation in which an imaging procedure needed to change hands, the result was probably predictable: serious under-utilisation of this exciting new non-invasive technique.

Peripheral angiography, however, historically belonged to radiology, and imaging of the carotid, renal and peripheral vessels simply became non-invasive procedures, performed in the radiology department and referred by vascular surgeons, physicians and neurosurgeons.

Cardiac magnetic resonance (CMR) imaging is the latest technique among the modalities used for imaging of the heart and great vessels. Huge research interest led to an enormous number of publications presenting a clear understanding of its absolute value, especially when assessing myocardial perfusion, viability and function in ischaemic heart disease. CMR is also very valuable in the diagnosis of the cardiomyopathies, arrhythmogenic conditions, tumours etc. There is unfortunately a growing trend for publications to be authored solely by cardiologists. In the USA, $37.7 \%$ of studies are by cardiologists, according to a survey published in the Journal of the American College of Radiology (JACR) in 2006, indicating a decreasing involvement by the radiology community. Despite the significant technical advances of the past 20 years, and growing research interest, relatively few cardiac MRI studies are performed by radiologists, compared with competing and often inferior tests. Alongside coronary CTA, cardiovascular MRI has become another orphan in our imaging armamentarium. The current economic climate worldwide as well as increasing pressures to curb the escalation in medical costs, are factors that clearly discourage the growth of CMR. It is furthermore not easy to prove to medical aid companies that a single, accurate and highly diagnostic - albeit expensive - modality would in many instances eliminate further additional and invasive tests.

\section{The future}

The problems outlined are not susceptible to easy resolution. However, as a first step, better communication between the parties involved would obviously help.

Renewed interest in cardiovascular imaging among radiologists in South Africa, and the possible establishment of a fellowship in cardiac imaging locally, would naturally assist in enhancing our credibility. Guidelines for CMR have been drawn up and task force criteria published. These need to be discussed among referring clinicians, radiologists and medical aid companies.

The argument against self-referral is a very valid one: it has undeniably led to escalating medical costs worldwide. According to a metaanalysis published in the JACR recently (July 2011), the estimated cost in the USA in 2006 of increased imaging from self-referral was $\$ 3.6$ billion. From 1996 to 2006, the total outpatient imaging rate by non-radiologists has increased by $71 \%$. To attribute the rise in cardiac imaging exclusively to self-referral would be a distortion of the facts, as the escalation in costs is largely due to burgeoning technical advances 


\section{EDITORIAL}

in recent years. This rise has furthermore paralleled a decline in the rate of death from heart disease as well as improvement in the quality of life for those living longer with heart disease. This is a fact often overlooked by governments and reimbursement companies. If we want to move to a proactive model of disease management, earlier and more advanced imaging is necessary, and government bodies and medical aid companies need to be involved in future discussions.

The unique situation necessitating diagnostic procedures to be performed by radiologists, which traditionally belonged in the catheterisation laboratory, needs to be understood by the radiology community, and more tolerance between the groups is necessary. The emphasis should be on finding a reasonable solution while steering away from a territorial dispute that will tend to result in total exclusion of the cardiologist's input in order to protect the radiologist's domain. Since cardiovascular disease is the leading cause of death worldwide, imaging of its core component - the heart - can hardly be regarded as unimportant by the radiology community.

\section{Conclusion}

Approximately 17 million people die every year from cardiovascular disease or stroke. South Africa has a very high incidence of ischaemic heart disease. We have access to superb diagnostic tools that enable us to play a pivotal role in the non-invasive diagnosis of heart disease. I believe that the time is ripe for renewed discussions between the roleplayers (the RSSA, cardiologists, radiologists and reimbursing companies) so as to elevate coronary CTA and CMR to the level where they belong: undoubtedly enormously valuable diagnostic tools that are currently shamelessly underutilised, to the detriment of all too many patients who have an enhanced chance of dying of a cardiovascular condition as a result of being investigated by inferior and/or invasive tests.

Why do we get passionate about imaging everything else, from cortical white matter down to the ligaments of the feet, and in the process bypass the heart? Why are we turning our backs on the largest pandemic in the

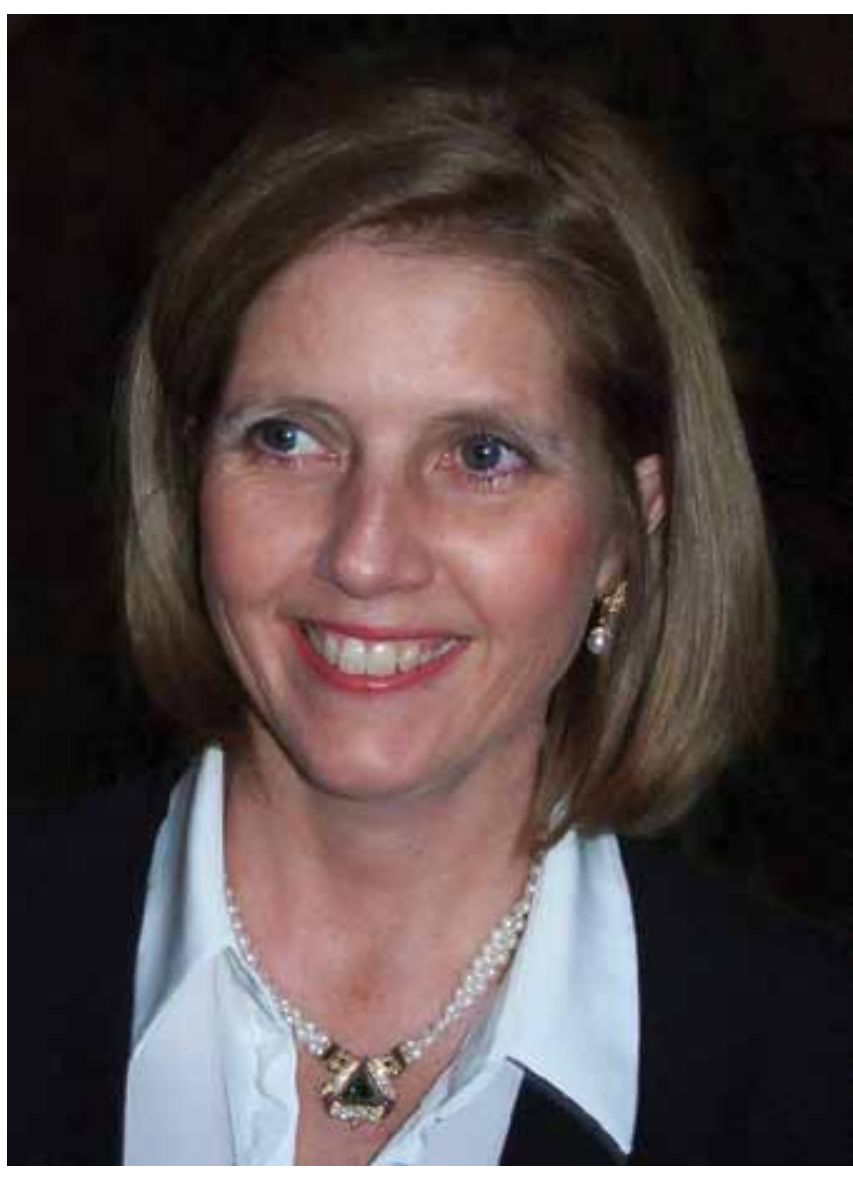

world? Are we too busy, too comfortable, too consumed with protecting our 'economic turf', or have we simply given up on matters of the heart?

\section{Leonie Scholtz}

Pretoria Heart Hospital

Pretoria

scholtzleonie@gmail.com 